\title{
Effect of Simultaneous Administration of Dihydroxyacetone on the Diffusion of Lawsone Through Various In Vitro Skin Models
}

\author{
Daniel J. Munt, ${ }^{1}$ Anne Grana, ${ }^{1}$ Martin Hulce, ${ }^{2}$ Ramon M. Fusaro, ${ }^{1,3,4}$ and Alekha K. Dash ${ }^{1,5,6}$
}

Received 18 March 2015; accepted 11 May 2015; published online 19 May 2015

\begin{abstract}
Unprotected sunlight exposure is a risk factor for a variety of cutaneous cancers. Topically used dihydroxyacetone (DHA) creates, via Maillard reaction, chemically fixed keratin sunscreen in the stratum corneum with significant protection against UVA/Soret radiation. When used in conjunction with naphthoquinones a naphthoquinone-modified DHA Maillard reaction is produced that provides protection across the UVB/UVA/Soret spectra lasting up to 1 week, resisting sweating and contact removal. The aim of this study was to examine a simplified version of this formulation for effect on UV transmission and to determine if penetration levels merit toxicity concerns. Permeability was demonstrated for freshly prepared DHA $(30 \mathrm{mg} / \mathrm{mL})$ and lawsone $(0.035 \mathrm{mg} / \mathrm{mL})$ alone and in combination using a side-by-side diffusion apparatus at $37^{\circ} \mathrm{C}$ over $48 \mathrm{~h}$ across shed snake skin and dermatomed pig skin. These samples were then examined for effectiveness and safety. Concentrations were determined by HPLC and UPLC monitored from 250-500 nm. Lawsone flux significantly decreased across pig skin $(20.8( \pm 4.8)$ and 0.09 $( \pm 0.1) \mathrm{mg} / \mathrm{cm}^{2} \mathrm{~h}$ without and with DHA, respectively) but did not change across shed snake skin in the presence of DHA. Significantly reduced lawsone concentration was noted in donor chambers of combined solutions. Damage was not observed in any skins. Darker coloration with greater UV absorbance was observed in skins exposed to the combined solution versus individual solutions. This study confirmed that combined DHA and lawsone provided effective blocking of ultraviolet light through products bound in keratinized tissue. DHA permeation levels in pig skin suggest further in vitro and in vivo study is required to determine the safety of this system.
\end{abstract}

KEY WORDS: cancer; dihydroxyacetone; FAMMM; naphthoquinone; skin.

\section{INTRODUCTION}

Cutaneous melanoma affects 21 of every 100,000 people in the USA each year: Approximately $2 \%$ of the population will develop melanoma at some point (1). Risk factors for developing cutaneous melanoma include environmental factors such as sun exposure, sun burns, and latitude as well as genetic factors such as amount of melanization of skin color (2-4). Having one or more family member with malignant melanoma(s) increases the risk two- to threefold. Melanoma is hereditary in approximately $10 \%$ of cases and fatal in approximately $12.4 \%$ of cases (1). Although survival rates have

${ }^{1}$ Department of Pharmacy Sciences, School of Pharmacy and Health Professions, Creighton University, Omaha, Nebraska, USA.

${ }^{2}$ Department of Chemistry, Creighton University, Omaha, Nebraska, USA.

${ }^{3}$ Departments of Preventive Medicine and Internal Medicine, Creighton University School of Medicine, Omaha, Nebraska, USA.

${ }^{4}$ Department of Internal Medicine, University of Nebraska School of Medicine, Omaha, Nebraska, USA.

${ }^{5}$ HLSB Room 109, 2500 California Plaza, Omaha, Nebraska 68178, USA.

${ }^{6}$ To whom correspondence should be addressed. (e-mail: adash@creighton.edu) increased due to advances in medicine, the incidence of cutaneous melanoma has steadily risen, having more than doubled since 1975 (1). Most cutaneous malignancies occur in two contexts. One group of individuals has spontaneous isolated non-hereditary melanomas, especially those with less pigmented skin (4). A second, smaller group is composed of families with the hereditary familial atypical multiple mole melanoma (FAMMM) syndrome (5). Both groups are induced by excessive exposures to UVB/A radiation from sunlight.

Lawsone (2-hydroxy-1,4-napthoquinone, also called hennotannic acid) is the primary coloring agent of red henna, a dye prepared from Lawsonia inermis leaves. It is used in henna tattooing, reverse tattooing, and as a hair dye (6). Lawsone has been found to have anti-oxidant, anti-microbial, anti-fungal, and anti-cancer properties as well as other properties (6-8). Lawsone is generally considered safe for topical application, but henna/henna extract is frequently paired with other materials such as $p$-phenylenediamine to enhance color which can result in dermatitis $(6,9)$.

1,3-Dihydroxyacetone (DHA) is a 3-carbon reducing sugar and the primary active ingredient of most sunless tanning products. Application of DHA to the skin causes a Maillard reaction in the stratum corneum, turning the skin a brown color which lasts until the stratum corneum is shed. It has been shown that the DHA Maillard reaction results in the 
production of UVA protective amorphous melanoidins causing it to be examined as a potential alternative to standard sunscreens (10-13). DHA is generally considered to be nontoxic and non-carcinogenic, but concerns have been raised over the production of free radicals and advanced glycylation endproducts (AGEs) associated with its reducing properties (14-18).

It is known that DHA interacts with lawsone, and it has been suggested that when DHA is used in combination with lawsone the resulting sunless tan may provide enhanced UVB/ A/Soret band protection compared to use of DHA alone (1923). This formulation has been examined clinically with respect to photoprotection in erythropoietic protophorphyria (24).

Clinical studies using Duosheild $1 \& 2$ (a binary spray system containing lawsone and DHA as active ingredients) from Rowell Labs have revealed that when DHA and naphthoquinones are applied to the skin as a simultaneous spray from separate containers, the topical application initiates the naphthoquinone modified DHA Maillard (NMDM) reaction overnight, over 4 to $6 \mathrm{~h}$, only in the metabolically inactive stratum corneum resulting in a chemically fixed keratin sunscreen to maintain continuous maximal UVB/A/Soret band photoprotection (UVB-SPF>18-24) (19-24). This dual spray application was to be applied once at bedtime followed by a shower to remove excess the following morning. Application on the first and second evening provides initial protection and coverage of missed spots. Future applications need only be applied two to three times per week. The keratin-fixed NMDM sunscreen within the stratum corneum cannot be removed by topical sweating, swimming, soap bathing, and contact with clothes. The photoprotection lasts a few days to 1 week and is lost eventually through normal desquamation from the skin's exfoliation process. This keratin-fixed sunscreen does not exist in the layers of the epidermis below the stratum corneum. Since it is lost by normal exfoliation, this keratin-fixed sunscreen has been shown to be safe and nontoxic $(19,20)$ by two long-term clinical studies supported by Rowell Laboratories. In these studies, numerous laboratory tests were done to establish that these patients did not exhibit any abnormal effects and demonstrated normal values for the tests that were done to meet US FDA standards for safe use by the public (25). Additionally, DHA alone has been shown to be non-carcinogenic in mice following a study in which DHA was painted onto the skin of mice for the life of the animal (15).

The aim of this study was to re-examine this approach and independently examine the permeability, absorption, and changes in the skin/stratum corneum. This was accomplished using a system consisting only of the active ingredients, lawsone and DHA, applied as a mixture created at the time of application in vitro through the use of the porcine and shed snake skin models. These models have been well documented for their correlation to human systems $(6,26-34)$ and are abundantly available. The shed snake skin model was chosen for these studies in our lab to examine the specific interactions of lawsone in the presence of DHA with the stratum corneum. This model provides a sturdy keratin-based substrate that can mimic chemical interactions with the stratum corneum. The lack of appendages (hair and pores) on the shed snake skin helps provide an intact membrane to isolate these interactions.
Due to observations of potential toxicity observed by other labs (14-18), we also wished to examine whole skin interactions to see how much if any of the drugs was likely to penetrate to the blood stream. For this, we chose to use dermatomed porcine skin as a preliminary model before testing on human skin models. For all diffusion studies, we used $3 \mathrm{~mL}$ donor and receiver chambers, larger than that typically used in these experiments with an extended exposure time in an attempt to simulate long-term continued exposure to the system. Our working hypothesis is that, when applied simultaneously as separate solutions, lawsone and dihydroxyacetone are sequestered primarily in the stratum corneum which minimizes systemic exposure and provides broad-spectrum reduction in UV transmission.

\section{MATERIALS AND METHODS}

\section{Reagents}

Lawsone was obtained commercially as a $97 \%$ powder (Sigma-Aldrich). Reagent-grade acetonitrile, DMSO, methanol, low melting temperature paraffin, paraformaldehyde, sodium phosphate monobasic, sodium phosphate dibasic, and $100 \mathrm{U} / \mathrm{mL}$ penicillin-streptomycin were obtained commercially from Fisher Scientific. DHA and 2-phenylethanol were obtained from Acros.

\section{Diffusion Studies}

\section{Shed Snake Skin Diffusion Studies}

Shed snake skin was obtained from the Henry Doorly Zoo (Omaha, NE); reticulated python skin was selected for these studies due to the large surface area with extensive portions of uniform skin allowing for more inclusive matched skin samples for testing. Skin was matched by color and scale size for each experiment. 2-Phenylethanol was selected as a size matched (to lawsone) diffusion control in the experiments to ensure that differences observed were not a result of natural variation between skin samples or changes in the porosity of the skins as a result of DHA interactions with skin proteins.

Lawsone, 2-phenylethanol, and DHA solutions were prepared immediately prior to exposure to diffusion chambers in $0.1 \mathrm{M} \mathrm{pH} 7.4$ phosphate buffer (PB). Lawsone and 2phenylethanol solutions were prepared to a final concentration of $0.035 \mathrm{mg} / \mathrm{mL}$ in PB, and DHA was prepared as a 30$\mathrm{mg} / \mathrm{mL}$ solution in $\mathrm{PB}$. The following groups comprised the experimental set for these experiments: 2-phenylethanol alone, 2-phenylethanol with DHA, lawsone alone, DHA alone, and lawsone with DHA. Data represent the average of three experiments each consisting of three cells $(n=9)$.

Prior to each experiment, matched portions of shed snake skin were allowed to equilibrate for $24 \mathrm{~h}$ in the PB. Following equilibration, snake skin was cut into $\sim 3 \times 3 \mathrm{~cm}$ squares and placed into a side-by-side diffusion apparatus with the exterior of the skin oriented to the donor chamber. First, the donor chamber was filled with $3 \mathrm{~mL} \mathrm{~PB}$, allowed to thermally equilibrate, and was examined for leaks after $10 \mathrm{~min}$. Then, the receiver chamber was filled with $3 \mathrm{~mL} \mathrm{~PB}$ and allowed to thermally equilibrate, and the apparatus was examined for leaks after $10 \mathrm{~min}$. Temperature was maintained at $37^{\circ} \mathrm{C}$ using 
a thermostated water jacket for the duration of the experiment. After equilibration and examination for leaks, the donor solvent was completely removed and replaced with $3 \mathrm{~mL}$ of drug solution. At 3-, 6-, 12-, 24-, and 48-h intervals, $150 \mu \mathrm{L}$ of sample was collected from each receiver chamber and replaced with $\mathrm{PB}$. At 0 and $48 \mathrm{~h}$, donor chamber samples also were collected. All collected samples were analyzed by HPLC. Snake skin was also retained for examination of optical properties.

\section{Porcine Skin Diffusion Studies}

Lawsone and DHA solutions were prepared immediately prior to exposure to diffusion chambers in diffusion solvent $(0.2 \mu \mathrm{m}$ filtered $0.1 \mathrm{M} \mathrm{PB}$ pH 7.4 supplemented with 100 units per $\mathrm{mL}$ penicillin-streptomycin solution). Lawsone was prepared to a final concentration of $0.035 \mathrm{mg} / \mathrm{mL}$, and DHA was prepared to a final concentration of $30 \mathrm{mg} / \mathrm{mL}$. The following groups comprised the experimental set for these experiments: lawsone alone, DHA alone, and lawsone with DHA. Temperature within the diffusion chambers was maintained at $37^{\circ} \mathrm{C}$ using a thermostated water jacket for the duration of the experiment.

A fresh frozen suckling pig was obtained, shaved, and the outer $0.5 \mathrm{~mm}$ of skin was removed using a Silvers skin graft knife. Skin samples were sorted by body area and by color. Matched skin was cut into $\sim 3 \times 3 \mathrm{~cm}$ squares and placed into a side-by-side diffusion apparatus with the exterior of the skin oriented to the donor chamber. First, the donor chamber was filled with $3 \mathrm{~mL} \mathrm{~PB}$, allowed to thermally equilibrate, and was examined for leaks after $10 \mathrm{~min}$. Then, the receiver chamber was filled with $3 \mathrm{~mL} \mathrm{~PB}$ and allowed to thermally equilibrate, and the apparatus was examined for leaks after $10 \mathrm{~min}$. After equilibration and examination for leaks, donor solvent was replaced with $3 \mathrm{~mL}$ of drug solution.

At 1-, 3-, 6-, 12-, 24-, and 48-h intervals, $100 \mu \mathrm{L}$ of sample was collected from each receiver chamber and replaced with diffusion solvent. At 0 and $48 \mathrm{~h}$, donor chamber samples also were collected. All collected samples were filtered and analyzed by UPLC. Skins were also retained for examination of optical properties. Data represent the average of three experiments each consisting of three cells $(n=9)$.

\section{HPLC Analysis of Lawsone, DHA, and 2-Phenylethanol}

Quantitative analysis of lawsone, DHA, and 2phenylethanol in the receiver and donor chambers for the shed snake skin experiments was performed using an HPLC method developed and validated by our lab according to the United States Pharmacopeia (USP) guidelines for linearity, accuracy, precision, specificity, and robustness. This method was carried out at room temperature using reverse phase HPLC using a photo-diode array UV detector from 230$400 \mathrm{~nm}$ and a flow rate of $0.8 \mathrm{~mL} / \mathrm{min}$ for $30 \mathrm{~min}$. A Shimadzu HPLC system was used consisting of a SCL-10Avp control unit, a SIL-10ADvp auto-injector, two LC-10ATvp pumps, a CTO-10ASvp column oven, and a SPD-M10Avp UV detector (Shimadzu, Kyoto, Japan) connected to a computer for data acquisition and processing (Shimadzu Class-VP version 7.2.1 build 22). Before analysis, all samples were filtered using 0.45 $\mu \mathrm{m}$ syringe filters. A $20-\mu \mathrm{L}$ aliquot was injected onto a Zorbax
SB-C $\mathrm{C}_{18}$ column $(5 \mu \mathrm{m}, 4.16 \times 150 \mathrm{~mm})$ (Agilent, Santa Clara, CA), and separation was achieved using a mobile phase consisting of 40:60 (\%,v/v) acetic acid (0.1 M)/acetonitrile. This method showed a detection limit of $0.12 \mathrm{ug} / \mathrm{mL}, 0.12 \mathrm{ug} /$ $\mathrm{mL}$, and $24 \mathrm{ug} / \mathrm{mL}$ for lawsone, 2-phenylethanol, and DHA, respectively. Standard solutions were prepared from 5-0.2 $\mu \mathrm{g}$ / $\mathrm{mL}$ for lawsone and 2-phenylethanol and $50-0.1 \mathrm{mg} / \mathrm{mL}$ for DHA, respectively, using PB. DHA standard curve was linear from 0.1 to $50 \mathrm{mg} / \mathrm{mL}$. Two linear standard curves $\left(R^{2}>0.999\right)$ were derived for each lawsone and 2-phenylethanol to cover all required concentration ranges from 5 to $0.016 \mathrm{mg} / \mathrm{mL}$ and from 0.016 to $0.2 \mu \mathrm{g} / \mathrm{mL}$. Dihydroxyacetone, lawsone, and 2phenylethanol were retained at $1.7,7.8$, and $7 \mathrm{~min}$, respectively.

\section{UPLC Analysis of Lawsone and DHA}

Quantitative analysis of lawsone and DHA in the receiver and donor chambers for the porcine skin experiments was performed using a UPLC method developed and validated by our lab according to the USP guidelines for linearity, accuracy, precision, specificity, and robustness. This method was carried out at room temperature using reverse phase UPLC using a photo-diode array UV detector from $230-400 \mathrm{~nm}$ and a flow rate of $0.5 \mathrm{~mL} / \mathrm{min}$ for $6 \mathrm{~min}$. A Waters Acquity $\mathrm{H}$ Class UPLC system was used consisting of a control unit, an autoinjector, a quaternary pump, a two-column preheated column manager, and a PDA detector (Waters, Milford, MA) connected to a computer for data acquisition and processing (Waters, Empower 2 release 5 build 2154). Before analysis, all samples were filtered using $0.2-\mu \mathrm{m}$ syringe filters. A $10-\mu \mathrm{L}$ aliquot was injected onto a Waters Acquity $\mathrm{BEH}-\mathrm{C}_{18}$ column (1.7 $\mu \mathrm{m}, 2.1 \times 50 \mathrm{~mm})$ (Waters, Milford, MA) and separation was achieved using a mobile phase consisting of 70:30 $(\%, v / v)$ acetic acid $(0.1 \mathrm{M}) /$ methanol. This method showed a detection limit of 0.06 and $12 \mathrm{ug} / \mathrm{mL}$ for lawsone and DHA, respectively. Standard solutions were prepared from $5-0.2 \mu \mathrm{g} / \mathrm{mL}$ for lawsone and $50-0.1 \mathrm{mg} / \mathrm{mL}$ for DHA. DHA standard curve was linear from 0.1 to $50 \mathrm{mg} / \mathrm{mL}$. Two linear standard curves $\left(R^{2}>0.999\right)$ were derived for each lawsone and 2phenylethanol to cover all required concentration ranges from 5 to $0.016 \mathrm{mg} / \mathrm{mL}$ and from 0.016 to $0.2 \mu \mathrm{g} / \mathrm{mL}$. Dihydroxyacetone and lawsone were retained at 0.3 and $1.7 \mathrm{~min}$, respectively.

\section{Examination of Optical Properties of Exposed Snake Skin}

Snake skin samples from diffusion studies were washed in diffusion solvent, stretched onto clean untreated glass microscope slides, and allowed to dry. After drying, skins were pressed between a second microscope slide and the mounted samples held in place physically using binder clips at both ends of the slides. Skins mounted in this manner were examined with respect to UV, fluorescence, cross-polarized, and transmitted visible light properties using vehicle-treated skins as controls.

UV exposure: mounted slides were grouped by experiment and placed on a Fotodyne Foto/UV 26 transilluminator emitting $312 \mathrm{~nm}$ UV light set on high intensity in a dark room, and then photographed using Polaroid 667 film on a 1-s exposure. 


\section{a}

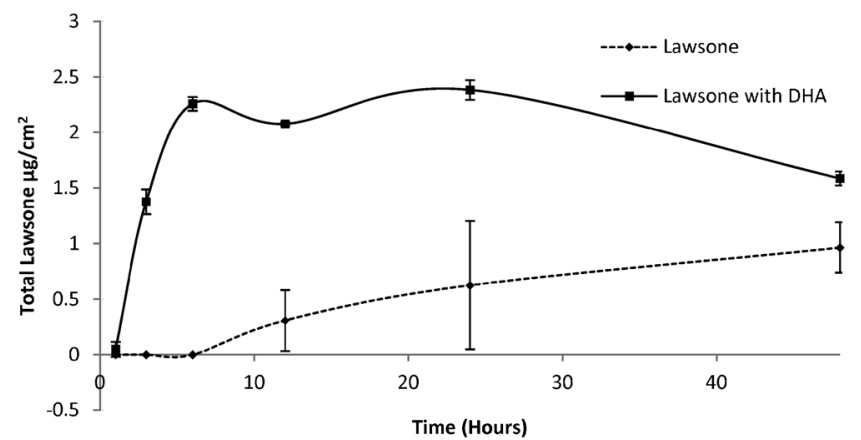

b

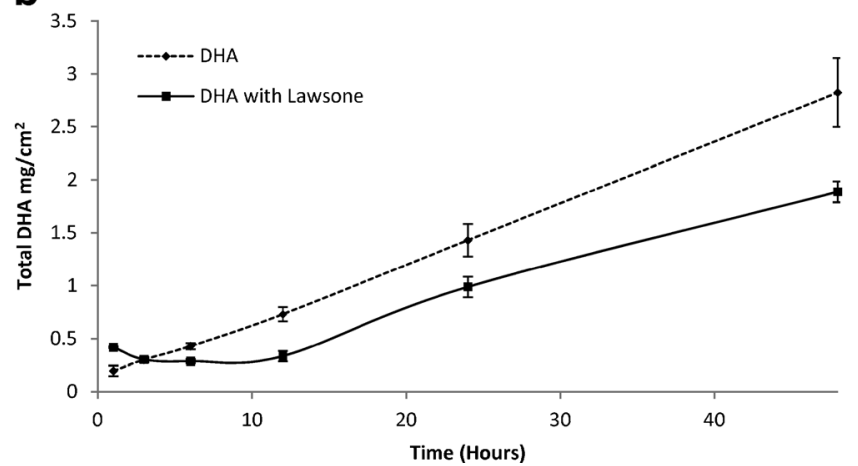

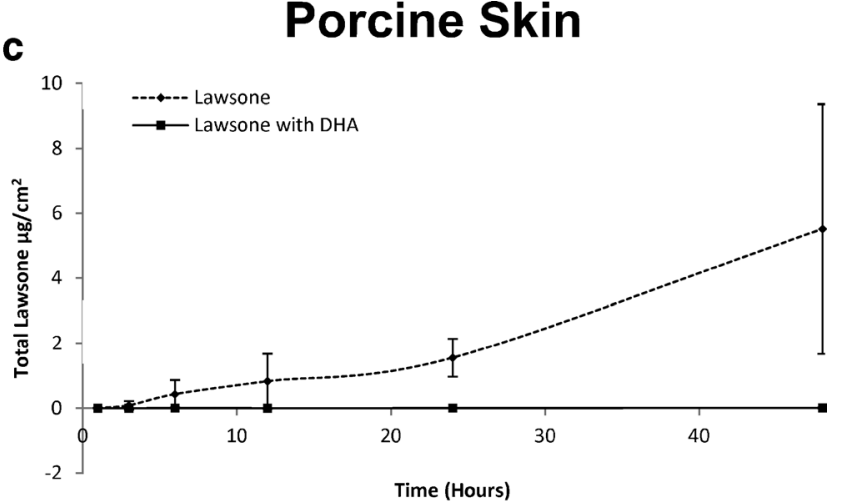

d

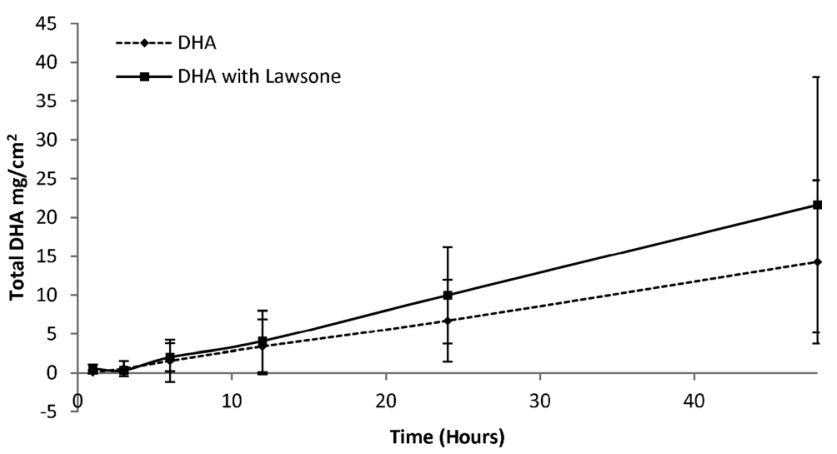

Fig. 1. Average cumulative amount of lawsone (a and $\mathbf{c})$ and DHA (b and $\mathbf{d})$ diffused through shed snake skin (a and $\mathbf{b})$ and porcine skin (c and d) in a side-by-side diffusion chamber per surface area over $48 \mathrm{~h}$ at $37^{\circ} \mathrm{C}$. Samples were prepared immediately prior to the experiment in phosphate buffer. Error bars represent standard deviations $(n=9)$

Fluorescence, cross-polarized, and transmitted light properties were all examined on a Leica DM2500M microscope with A4, L5, and N3 fluorescent filter cubes, DFC320 camera, and analyzed using Leica Application Suite 2.3.2 R1, and Image J software. Filters A4, L5, and N3 represent filters 3, 2 , and 1; have UV, blue, and green excitation filters; and blue, green, and red emission filters, respectively. In all experiments, a background level (for untreated skin) was obtained and exposure times/magnification was maintained between samples. Samples that resulted in over- or underexposure at the background level were examined to determine the exposure times for those samples that resulted in apparent equivalent overall brightness as compared to blank. These values were then collated by measuring the actual brightness using ImageJ software for each exposure.

For UV absorption studies, skin was mounted into a Biotek "Take3" slide and UV absorbance from 230-400 nm was measured from 8 points on each snake skin from the study in the treated area using a Biotek Synergy H1 microplate reader. The absorbances from these points were averaged to form the final absorption for each wavelength which was then plotted to form an average absorption curve. Results were plotted and area under the curve (AUC) was determined from 230 to $400 \mathrm{~nm}$ and from 230 to $360 \mathrm{~nm}$ as well as baseline (blank) subtracted values.

\section{Examination of Optical Properties of Exposed Porcine Skin}

Porcine skin retained from diffusion studies was examined with respect to UV, fluorescence, cross-polarized, and transmitted light properties using the same methodologies as for the snake skin. Due to the thickness of the samples, they were not examined for UV absorbance.

Table I. Amount of Lawsone in the Donor Chamber over $48 \mathrm{~h}$

\begin{tabular}{|c|c|c|c|}
\hline & Amount at $0 \mathrm{~h}(\mu \mathrm{g} \pm \mathrm{SD})$ & Amount at $48 \mathrm{~h}(\mu \mathrm{g} \pm \mathrm{SD})$ & Percent decrease in amount at $48 \mathrm{~h}^{\mathrm{a}}$ \\
\hline Through shed snake skin & $94.7 \pm 1.6$ & $87.8 \pm 3.1$ & $7.2 \pm 2.5$ \\
\hline Through shed snake skin with DHA & $85.5 \pm 5.6$ & $0.6 \pm 0.1$ & $99.3 \pm 3.3^{\mathrm{b}}$ \\
\hline Through porcine skin & $108.8 \pm 13.9$ & $76.4 \pm 7.6$ & $29.8 \pm 9.9$ \\
\hline Through porcine skin with DHA & $17.7 \pm 4.3$ & $4.8 \pm 1.4$ & $75.8 \pm 16.1^{\mathrm{b}}$ \\
\hline
\end{tabular}

Total average quantity of drug in the donor chambers of side-by-side diffusion apparatus $(n=9)$

$S D$ standard deviation, $D H A$ dihydroxyacetone

${ }^{a}$ Decrease in donor chamber quantities normalized to percentages to reflect differences from initial quantities

${ }^{b}$ Significant $(P<0.01)$ increase as compared to solution without DHA by Students 2 tailed $t$ test 


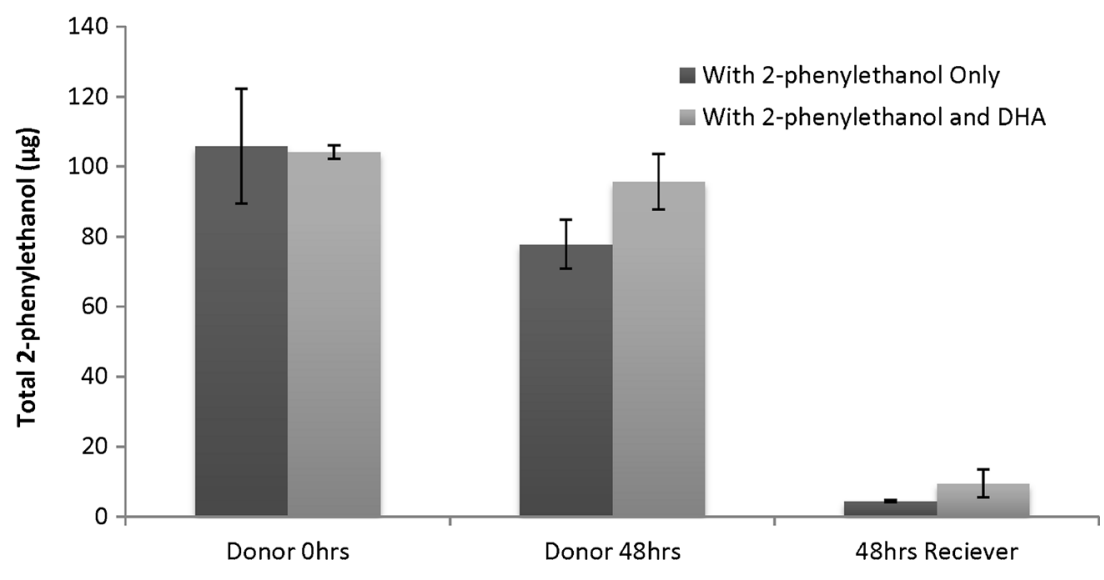

Fig. 2. Average amount of 2-phenylethanol (control) in the donor chamber at time zero and after $48 \mathrm{~h}$ of diffusion and the average amount of 2-phenylethanol in the receiver chamber after $48 \mathrm{~h}$. Samples were prepared immediately prior to the experiment in the presence and absence of DHA in phosphate buffer and allowed to diffuse through shed snake skin for $48 \mathrm{~h}$ at $37^{\circ} \mathrm{C}$ in a side-by-side diffusion chamber. Error bars represent standard deviations $(n=9)$

\section{Porcine Skin Microtomy}

In order to examine the penetration of lawsone/DHA and examine tissue for any potential damage, porcine skins used in the diffusion study were selected, fixed in $4 \%(\mathrm{w} / \mathrm{v})$ paraformaldehyde, embedded in paraffin, sectioned at $7 \mu \mathrm{m}$, and stained with hematoxylin and eosin. These sections were then examined under the Leica DM2500M microscope using transmitted light.

\section{Data Analysis}

Flux was calculated as the amount passing through the membrane per unit time per unit surface area of the membrane for each chamber then averaged and presented plus and minus standard deviation for the study $(n=9)$. For UV absorbance curves, the area under the curve was calculated using Image J software and compared as a percentage of the blank AUC. Oversaturated wavelengths were given a value of 4 in order to be plotted. Values for all quantified data were examined using a two-tailed Student's $t$ test and were considered significant if the calculated $P$ value was less than 0.05 .

\section{RESULTS}

The presence of DHA results in a significant $(P<0.05)$ initial increase in the diffusion of lawsone across the snake skin in PB. Diffusion begins to decrease in 12-24 h (Fig. 1a).

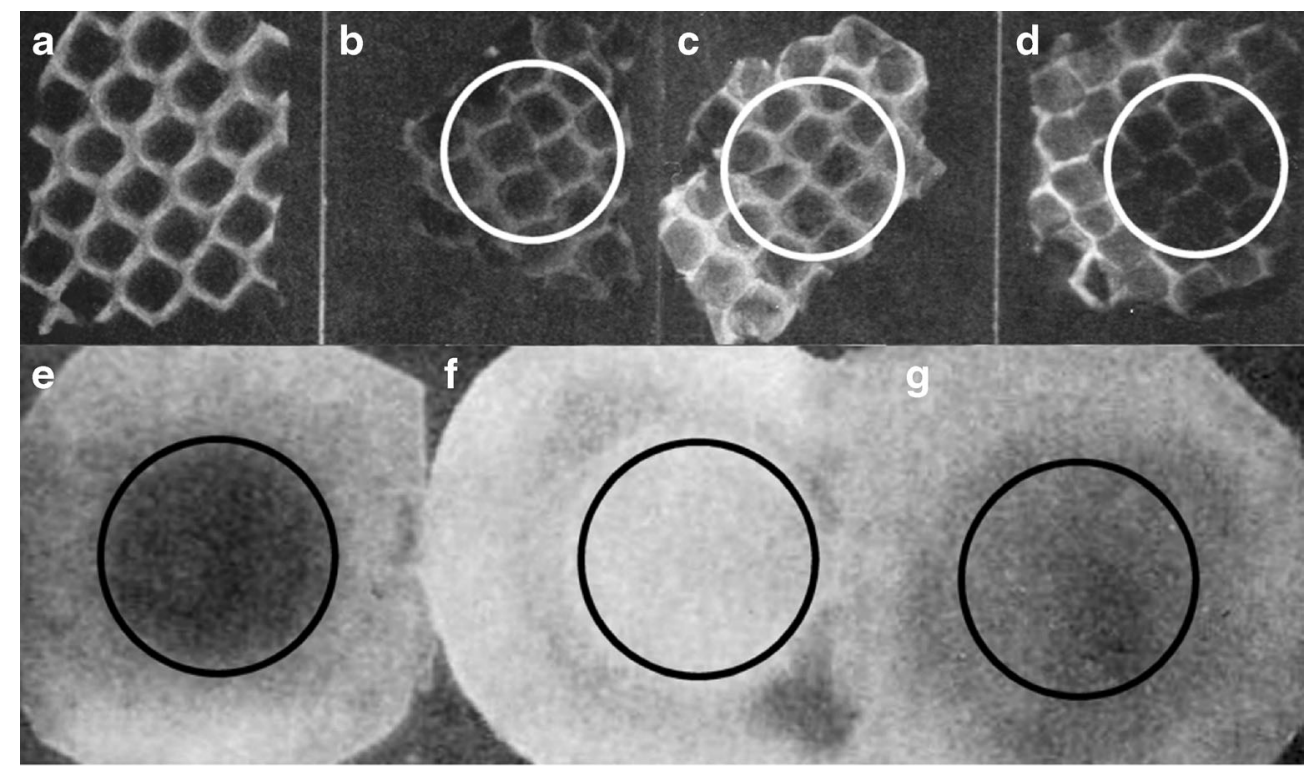

Fig. 3. Representative UV photographs of skin samples from shed snake skin (a-d) and porcine skin (e-g): blank (a), treated with lawsone only (b and $\mathbf{g}$ ), DHA only (c and f), or lawsone with DHA (d and e). Following diffusion studies, skins were washed in phosphate buffer, pressed between microscope slides, dried at room temperature and then photographed on a Fotodyne Foto/UV 26 transilluminator emitting $312 \mathrm{~nm}$ UV (UVB) 
There is no significant difference in the overall flux of lawsone across the snake skin in the absence or presence of DHA 0.13 $( \pm 0.04)$ and $0.11( \pm 0.08) \mu \mathrm{g} / \mathrm{cm}^{2} \mathrm{~h}$, respectively, by $72 \mathrm{~h}$. The diffusion of DHA across the snake skin is not significantly affected (Fig. 1b) by lawsone. Examining the donor chambers, a significant $(P<0.01)$ decrease in the quantity of lawsone in the presence of DHA as compared to lawsone alone was observed as shown in Table I. The diffusion of the control compound (2-phenylethanol) was not affected $(P>0.30)$ by the presence of DHA (Fig. 2).

The presence of DHA results in a significant $(P<0.05)$ decrease in the flux of lawsone across porcine skin, which shows little detectable flux by $48 \mathrm{~h}$ in the presence of DHA (Fig. 1c) registering $20.8( \pm 4.8)$ and $0.09( \pm 0.1) \mu \mathrm{g} / \mathrm{cm}^{2} \mathrm{~h}$ in the absence and presence of DHA, respectively. However, the diffusion of DHA across porcine skin is not significantly $(P>0.5)$ affected (Fig. 1d) $0.5 \pm 0.4$ and $1.6 \pm 1.9 \mathrm{mg} / \mathrm{cm}^{2} \mathrm{~h}$ for DHA and DHA with lawsone, respectively. Examining the donor quantities, a significant $(P<0.01)$ drop in the amount of lawsone in the presence of DHA as compared to lawsone alone was observed as shown in Table I.

Qualitative examination of UV exposure photography of the post-diffusion shed snake skin (Fig. 3a-d) and porcine skin (Fig. 3e-f) showed an overall UV transmission quenching in samples treated with lawsone and a combination of lawsone and DHA (Fig. 4b, g and d, e, respectively) as compared to vehicle- and DHA-treated skin (Fig. 3a, c, f). The greatest reduction was in samples exposed to a combination of lawsone and DHA. Samples that had been treated with DHA alone did not show significant UV quenching (Fig. 3c, f).

Quantitative UV absorbance studies verified the results observed in UV exposure studies (Fig. 4). Samples treated with lawsone and DHA had significantly $(P<0.05)$ greater broad spectrum UV absorbance, 221.57\% $( \pm 18.01)$, greater than lawsone alone. DHA alone had lower UV absorbance than the blank sample and so was not quantified.

Microscopic analysis of post-diffusion snake skin and porcine skin samples showed little change in transmitted light. In comparison to snake skin treated with only PB (Fig. 5a), lawsone treatment (Fig. 5b) showed no apparent change while DHA alone increased the transmission of polarized light (Fig. 5c). The combination of lawsone and DHA decreased the transmission of polarized light (Fig. 5d) through shed snake skin. In the porcine skin (Fig. 5e-g), all samples resulted in an apparent decrease in polarized light transmission compared to blank. Sectioned post-diffusion porcine skin (Fig. 6) did not show coloration below the stratum corneum. Additionally, there was little change in surface morphology of sectioned porcine skin and there were no physical indications of tissue damage in any of the samples.

Levels of snake skin fluorescence were determined qualitatively by observing emissions over a fixed exposure time, and were confirmed using variable exposure to replicate overall brightness (Fig. 7). Post-diffusion snake skin showed little change when DHA was used alone. Use of lawsone alone (Fig. 7i-1) reduced fluorescence on all filters, producing $78 \%$ of the emissions observed on blank for filter 1 and no detectable emissions at the standard exposure time for filters 2 and 3. When exposure times were increased for these filters by 7.3 and $10.2 \mathrm{~s}$, respectively, 69 and $96 \%$ of the corresponding blank fluorescence was observed. The use of a combination of lawsone and DHA (Fig. $7 \mathrm{~m}-\mathrm{p}$ ) resulted in a greater decrease in fluorescence, producing $14 \%$ of the fluorescence of a blank for filter 1 and no detectable emissions at the standard exposure time for filters 2 and 3 . When exposure times were increased for these filters by 26.1 and $28.4 \mathrm{~s}$, respectively, 27 and $76 \%$ of the corresponding blank fluorescence was observed.

\section{DISCUSSION}

The diffusion studies here show an overall decrease in the flux of lawsone across the porcine skin and an overall lack of change in flux of lawsone through the snake skin in the presence of DHA. Moreover, it shows a significant loss of lawsone within both donor compartments, suggesting that in the presence of DHA lawsone is being sequestered within the stratum corneum and the upper epidermis. This loss is greater in the

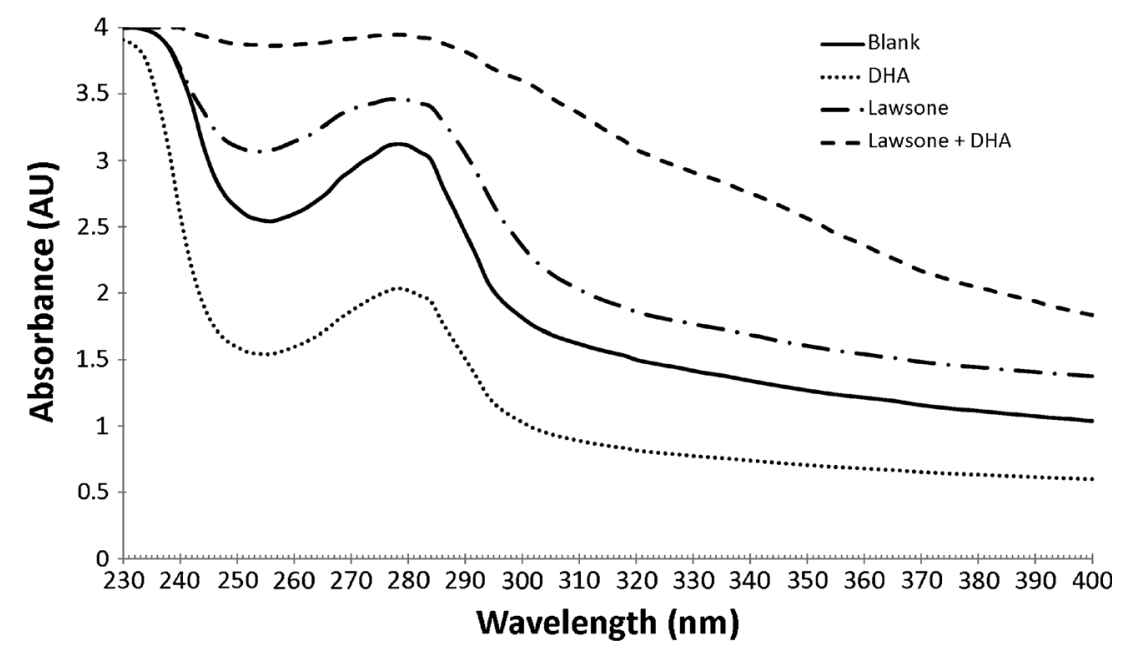

Fig. 4. Averaged UV absorbance spectra of snake skin samples from $230-400 \mathrm{~nm}$ in 2-nm steps $(n=8)$. Post-diffusion snake skins were washed in phosphate buffer, dried at room temperature, and dissected to individual scales which were placed in the individual microliter sample slide of a Take3 plate, pressed, and absorbance was read on a Biotek Synergy $\mathrm{H} 1$ reader. Individual points were averaged from eight samples 


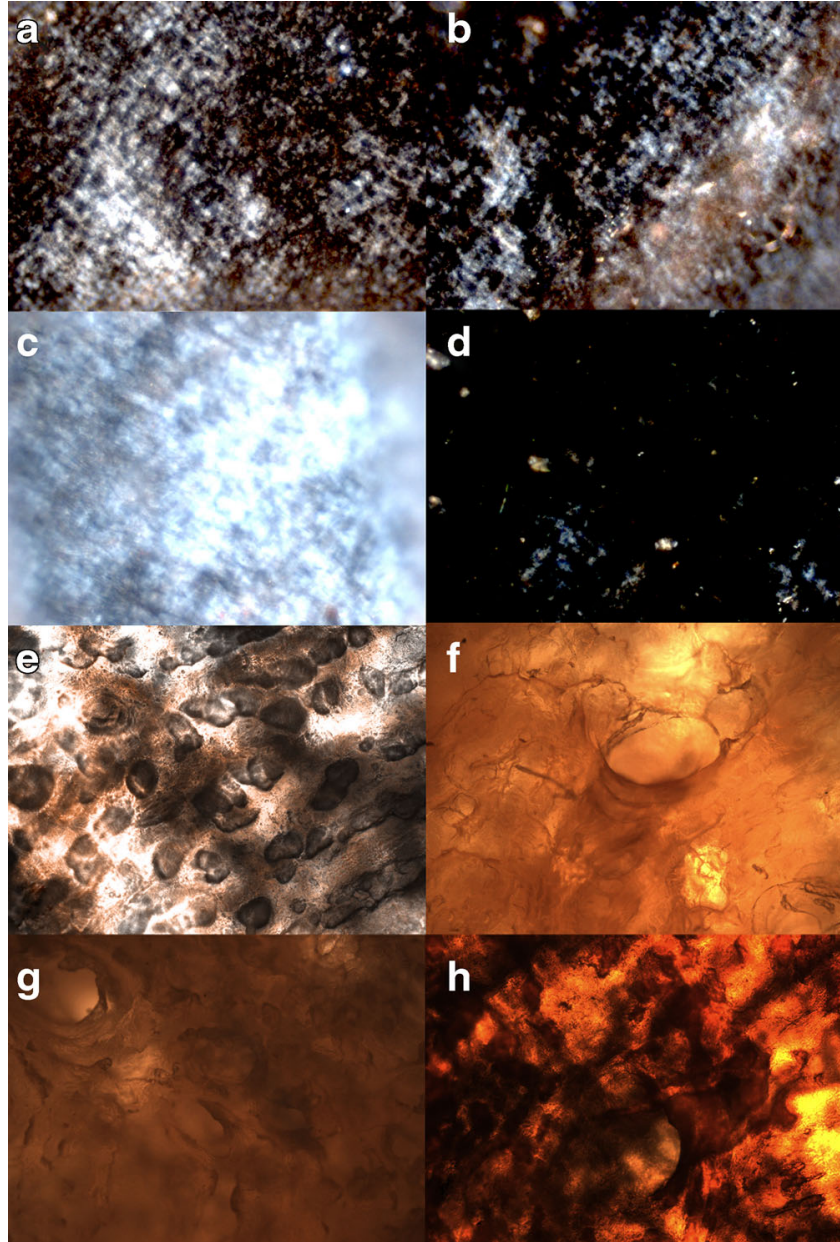

Fig. 5. Representative polarized light microscopy of shed snake skin (a-d) and porcine skin (e-h) treated with blank (a and e), lawsone only (b and f), DHA only (c and $\mathbf{g}$ ), or lawsone and DHA (d and $\mathbf{h}$ ). Following diffusion studies, skins were washed in phosphate buffer, pressed between microscope slides, dried at room temperature, and then examined under cross-polarized light

shed snake skin, and the overall transport of lawsone is lower in the shed snake skin. The thickened keratin of the shed snake skin adds to the suggestion that the lawsone is primarily being sequestered in within the stratum corneum. This activity may be sufficient to prevent the lawsone from diffusing far enough through the skin to reach the blood stream. Additionally, we see that the overall transport of DHA is not significantly altered by the presence of lawsone, in large part because the DHA is provided in excess for these experiments. The overall transport of DHA is also significantly greater in the porcine skin samples; this may be in part due to a thinner keratin layer of the porcine skin stratum corneum. As live skin was not used, this may also be due to the breakdown of sugars within the tissue producing artifacts. Microscopic examination revealed exposure of the tissue to the instant mixture or individual solutions does not cause any apparent direct damage to the stratum corneum or underlying tissue over $48 \mathrm{~h}$ of continuous exposure, as would be indicated by shedding or thinning of the stratum corneum or underlying tissue, or by holes, tears, or disrupted cell structure in the underlying tissue. The lack of damage to the stratum corneum in response to

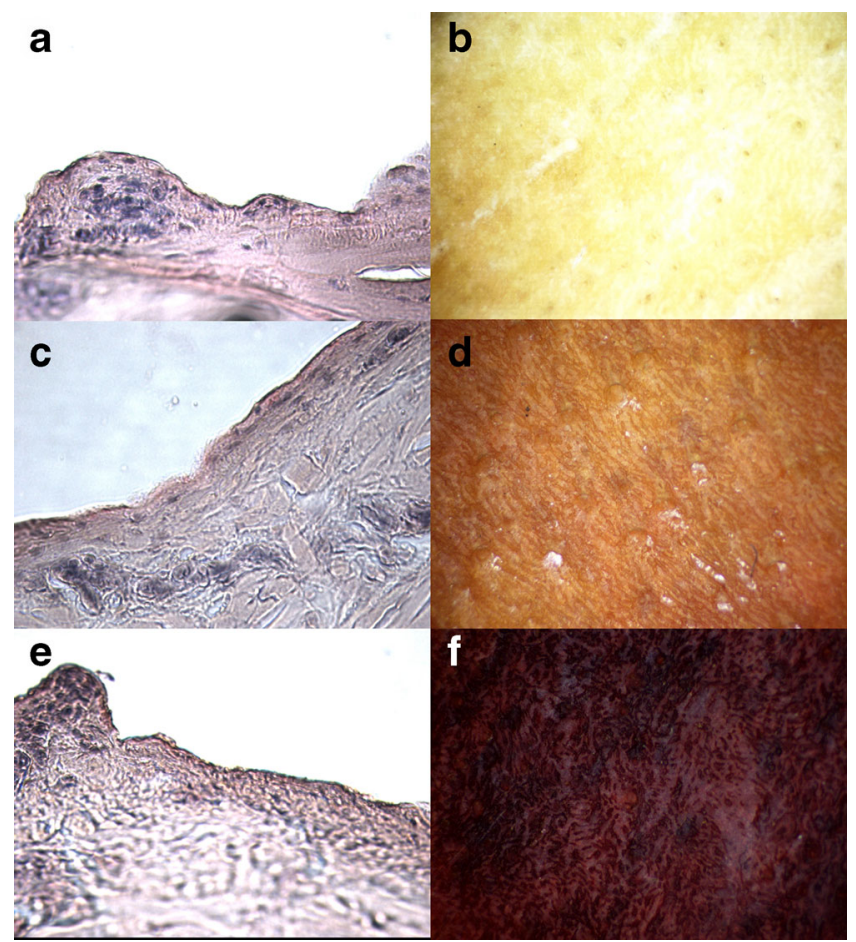

Fig. 6. Representative visible light microscopy of porcine skin sectioned at $\times 500$ magnification $(\mathbf{a}, \mathbf{c}, \mathbf{e})$ and grossly at $\times 50$ magnification $(\mathbf{b}, \mathbf{d}, \mathbf{f})$ for samples treated with DHA only $(\mathbf{a}, \mathbf{b})$, lawsone only $(\mathbf{c}, \mathbf{d})$, and a combination of lawsone and DHA $(\mathbf{e}, \mathbf{f})$. For sections following diffusion studies, skins were washed in phosphate buffer prior to embedding in paraffin and sectioning at $7 \mu \mathrm{m}$. For gross microscopy following diffusion studies, skins were washed in phosphate buffer, pressed between microscope slides, dried at room temperature, and photographed

DHA is supported also by the lack of change observed in the diffusion of the control 2-phenylethanol through the shed snake skin.

It is known that AGEs may be produced during the DHA Maillard reaction; within the stratum corneum, the amadori products (ketone rearrangements of Schiff bases formed as intermediates in the production of AGEs and melanoidins) are recycled into the reaction resulting in the ultimate formation of melanoidins (35). If the reaction occurs deeper than the dead stratum corneum, amadori products may be produced that are not recycled into the production of melanoidins resulting in the formation of AGEs which may pose oxidative stress risks depending upon the specific AGEs involved and may ultimately produce a problem in vivo $(35,36)$. Jung et al. reported the production of free radicals when DHA was used on live keratinocytes, suggesting a possible downfall of this approach if significant portions of the formulation reach live tissue (18). Our work, however, is using a lower dose of DHA than the smallest dose used in Jung's work (3\% $w / v$ versus $5 \% w / v)$. Jung's paper suggests an inverse logarithmic relationship between concentration and free radical production; extrapolating from the numbers in Jung's paper the dose used in our work would produce only a $0.05 \%$ increase in free radicals which would return to basal levels following within $20 \mathrm{~min}$. AGE production was also suggested as a potential problem where test concentrations ranged from 10 to $100 \mathrm{mM} \mathrm{DHA}$; the total 


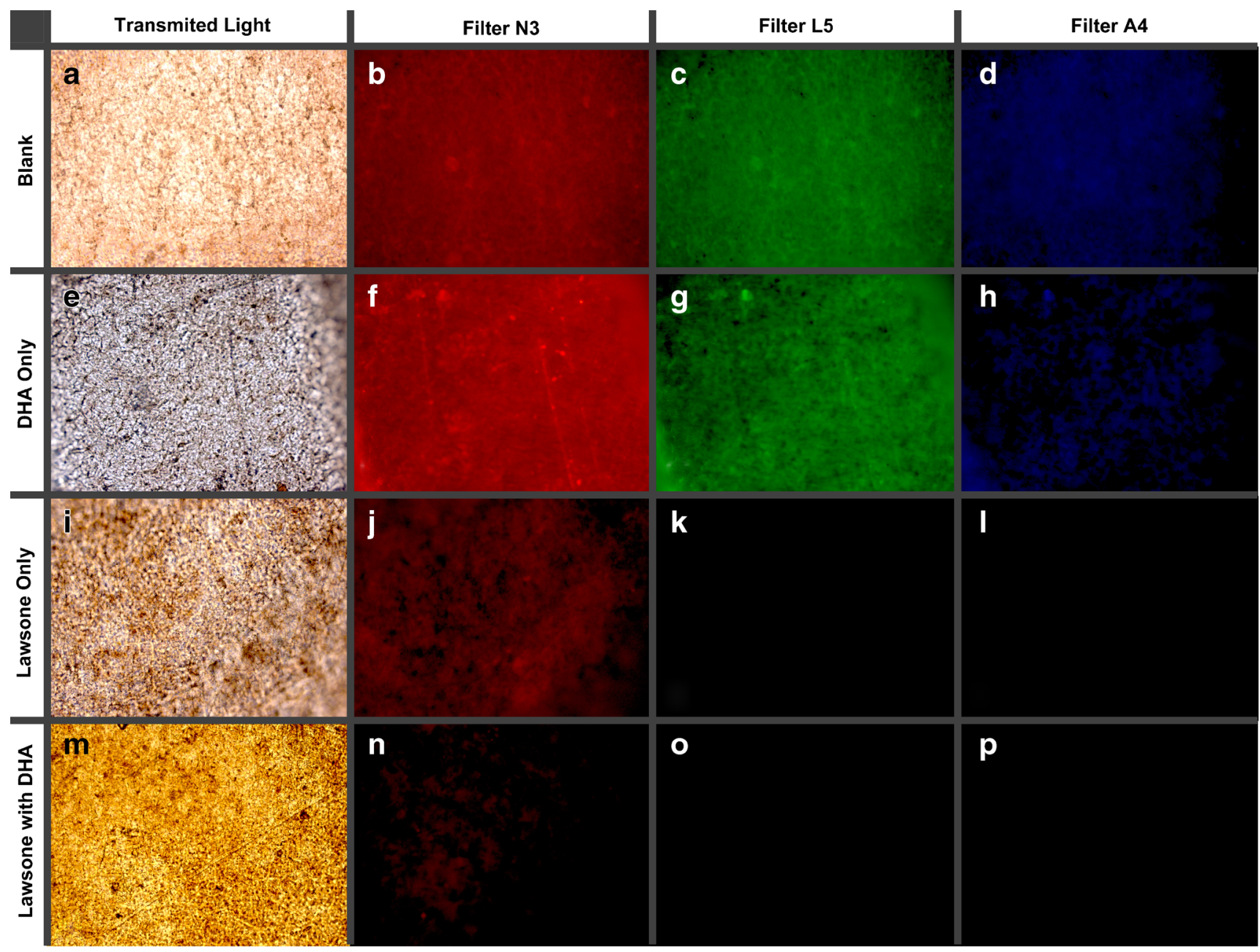

Fig. 7. Representative fluorescent light microscopy of snake skin treated with buffer only (a-d), treated with DHA (e-h), treated with lawsone $(\mathbf{i}-\mathbf{l})$, and treated with a combination of lawsone and DHA $(\mathbf{m}-\mathbf{p})$. Following diffusion studies, samples were washed in phosphate buffer, pressed between microscope slides, dried at room temperature, and examined. Each sample was examined under transmitted light (a, e, i, m), and using three fluorescent filters N3 (b, f, j, $\mathbf{n})$, L5 $(\mathbf{c}, \mathbf{g}, \mathbf{k}, \mathbf{o})$, and A4 $(\mathbf{d}, \mathbf{h}, \mathbf{l}, \mathbf{p})$

DHA to penetrate the membrane falls within this range in both cases (17). With respect to the shed snake skin, these values lay at the lower end where no effect was demonstrated. With respect to the dermatomed porcine skin, the concentration of DHA after $48 \mathrm{~h}$ of penetration was approximately 52-77 $\mathrm{mM}$, suggesting potential for AGE formation with the formulation in its current form. Our findings when looked at in the light of previous studies into the toxicity of DHA suggest that our formulation would likely not result in free radical problems though it may result in AGE formation which may or may not have an impact in vivo. To confirm this, future examinations will have to be done on the free radical production and in vitro and in vivo toxicity within our formulation.

The observed changes to the porcine and snake skins included an overall darkening of the skin as well as a broad spectrum increase in the absorbance of ultraviolet radiation, which correlates to a decrease in UV transmittance. This broad spectrum reduction UV transmittance could translate to broad spectrum UV protection. The overall decrease in polarized light transmission suggests that the products remaining within the skin exist as an irregular structure yet throughout the keratinized tissue. Coloration changes in sectioned porcine skin also confirm the absence of significant drug bound below the stratum corneum. As it is the result of a chemical interaction within the stratum corneum of the skin, this theoretically protective UV screen could continue to provide protection for a few days to a week, until the stratum corneum is shed. By reducing the effective exposure of those with limited natural photoprotection to high levels of sunlight, such protection could be of great benefit to persons with familial melanoma or to those who would otherwise develop non-hereditary melanomas and other skin cancers (20-24). Additionally, this semi-permanent sunscreen could also be useful in situations where frequent and prolonged exposure to direct sunlight is otherwise unavoidable and where the compliance to the regular application of sunscreen is likely to be low.

\section{CONCLUSION}

This study confirmed that when used in combination, DHA and lawsone provided effective blocking of ultraviolet 
light when applied to the skin surface. This protection and coloration appears to be due to the products of NMDM bound within the stratum corneum. However, the level of DHA that penetrated the porcine skin suggests that further in vitro and in vivo study is required to determine if $\mathrm{AGE}$ and free radical production is significant enough in both the basic solution and in the final formulation to merit concerns with the safety of this system.

\section{ACKNOWLEDGMENTS}

This research was partially funded by generous financial assistance from Creighton University.

\section{REFERENCES}

1. Howlader N, Noone AM, Krapcho M, Garshell J, Neyman N, Altekruse SF, Kosary CL, Yu M, Ruhl J, Tatalovich Z, Cho H, Mariotto A, Lewis DR, Chen HS, Feuer EJ, Cronin KA (eds). SEER Cancer Statistics Review, 1975-2010, National Cancer Institute. Bethesda, MD, http://seer.cancer.gov/csr/1975_2010/, based on November 2012 SEER data submission, posted to the SEER web site, April 2013.

2. Caini S, Gandini S, Sera F, Raimondi S, Fargnoli MC, Boniol M, et al. Meta-analysis of risk factors for cutaneous melanoma according to anatomical site and clinic-pathological variant. Eur J Cancer. 2009;45:3054-63.

3. Gandini S, Sera F, Cattaruzza MS, Pasquini P, Picconi O, Boyle $\mathrm{P}$, et al. Meta-analysis of risk factors for cutaneous melanoma: II. Sun exposure. Eur J Cancer. 2005;41:45-60.

4. Gandini S, Sera F, Cattaruzza MS, Pasquini P, Zanetti R, Masini $\mathrm{C}$, et al. Meta-analysis of risk factors for cutaneous melanoma: III. Family history, actinic damage and phenotypic factors. Eur J Cancer. 2005;41:2040-59.

5. Fusaro RM, Lynch HT, Kimberling WJ. Familial Atypical Multiple Mole Melanoma Syndrome (FAMMM). Arch Dermatol. 1983;119(1):2-3.

6. Semwal RB, Semwal DK, Combrinck S, Cartwright-Jones C, Viljoen A. Lawsonia inermis L. (henna): ethnobotanical, phytochemical and pharmacological aspects. J Ethnopharmacol. 2014;155:80-103.

7. Pradhan R, Dandawate P, Vyas A, Padhye S, Biersack B, Schobert R, et al. From body art to anticancer activities: perspectives on medicinal properties of henna. Curr Drug Targets. 2012;13(14):1777-98.

8. Kapadia GJ, Rao GS, Sridhar R, Ichiishi E, Takasaki M, Suzuki $\mathrm{N}$, et al. Chemoprevention of skin cancer: effect of Lawsonia inermis L. (Henna) leaf powder and its pigment artifact, lawsone in the Epstein- Barr virus early antigen activation assay and in two-stage mouse skin carcinogenesis models. Anticancer Agents Med Chem. 2013;13(10):1500-7.

9. Al-Shobaili HA, El-Bassouny DR. The ultrastructural effects of long-term use of henna on the albino rat skin. Histol Histopathol. 2014;29(3):333-42.

10. DeLeo V. Sunscreen use in photodermatosis. Dermatol Clin. 2006;24:27-33.

11. Armas LA, Fusaro RM, Sayre RM, Huerter CJ, Heaney RP. Do melanoidins induced by topical $9 \%$ dihydroxyacetone sunless tanning spray inhibit vitamin d production? A pilot study. Photochem Photobiol. 2009;85(5):1265-6.

12. Petersen AB, Na R, Wulf HC. Sunless skin tanning with dihydroxyacetone delays broad-spectrum ultraviolet photocarcinogenesis in hairless mice. Mutat Res. 2003;542(1-2):129-38.

13. Faurschou A, Wulf HC. Durability of the sun protection factor provided by dihydroxyacetone. Photodermatol Photoimmunol Photomed. 2004;20(5):239-42.

14. Goldman L, Blaney D, Goldman J. Topical therapy with dihydroxyacetone. Acta Dermatol Venereol. 1960;40:500-3.
15. Akin FJ, Marlowe E. Non-carcinogenicity of dihydroxyacetone by skin painting. J Environ Pathol Toxicol Oncol. 1984;5(45):349-51.

16. Seneviratne C, Dombi GW, Liu W, Dain JA. In vitro glycation of human serum albumin by dihydroxyacetone and dihydroxyacetone phosphate. Biochem Biophys Res Commun. 2012;417(2):817-23.

17. Petersen AB, Wulf HC, Gniadecki R, Gajkowska B. Dihydroxyacetone, the active browning ingredient in sunless tanning lotions, induces DNA damage, cell-cycle block and apoptosis in cultured HaCaT keratinocytes. Mutat Res. 2004;560(2):173-86.

18. Jung K, Seifert M, Herrling T, Fuchs J. UV-generated free radicals (FR) in skin: their prevention by sunscreens and their induction by self-tanning agents. Spectrochim Acta A Mol Biomol Spectrosc. 2008;69:1423-8.

19. Rice EG. Dihydroxyacetone napthoquinone protection against photosensitivity. Dermatol J. 1976;153:38-43.

20. Sletten IW, Fusaro RM, Runge WJ. A new topical spray agent to protect patients on chlorpromazine from sunlight. Am J Psychiatry. 1963;119:991-2.

21. Fusaro RM, Johnson JA. Photoprotection of patients sensitive to short and/or long ultraviolet light with dihydroxyacetone/ napthoquinone. Dermatologica. 1974;148:224-7.

22. Fusaro RM, Rice EG. The Maillard reaction for sunlight protection. Ann N Y Acad Sci. 2005;1043:174-83.

23. Fusaro RM, Johnson JA. Protection against long ultraviolet and/ or visible light with topical dihydroxyacetone. Implications for the mechanism of action of the sunscreen combination, dihydroxyacetone/naphthoquinone. Dermatologica. 1975;150(6):346-51.

24. Fusaro RM, Runge WJ. Erythropoietic protoporphyria. IV. Protection from sunlight. Br Med J. 1970;1(5698):730-1.

25. Department of Health, Education, and Welfare. Food and Drug Administration. 21 CFR Part 352. Sunscreen drug products for over-the-counter human use: establishment of a monograph; notice of proposed rulemaking. Fed Regist. 1978;43(166):38206-69.

26. Balogh TS, Pedriali CA, Gama RM, de Oliveira Pinto CA, Bedin $\mathrm{V}$, Villa RT, et al. Study of sunless tanning formulas using molted snake skin as an alternative membrane model. Int J Cosmet Sci. 2011;33(4):359-65.

27. Itoh T, Xia J, Magavi R, Nishihata T, Rytting JH. Use of shed snake skin as a model membrane for in vitro percutaneous penetration studies: comparison with human skin. Pharm Res. 1990;7(10):1042-7.

28. Craane-van Hinsberg WH, Verhoef JC, Bax LJ, Junginger HE, Boddé HE. Role of appendages in skin resistance and iontophoretic peptide flux: human versus snake skin. Pharm Res. 1995;12(10):1506-12.

29. Ngawhirunpat T, Panomsuk S, Opanasopit P, Rojanarata T, Hatanaka T. Comparison of the percutaneous absorption of hydrophilic and lipophilic compounds in shed snake skin and human skin. Pharmazie. 2006;61(4):331-5.

30. Benech-Kieffer F, Wegrich P, Schwarzenbach R, Klecak G, Weber T, Leclaire $\mathrm{J}$, et al. Percutaneous absorption of sunscreens in vitro: interspecies comparison, skin models and reproducibility aspects. Skin Pharmacol Appl Ski Physiol. 2000;13(6):324-35.

31. Gupta VK, Zatz JL, Rerek M. Percutaneous absorption of sunscreens through micro-yucatan pig skin in vitro. Pharm Res. 1999;16(10):1602-7.

32. Andrews SN, Jeong E, Prausnitz MR. Transdermal delivery of molecules is limited by full epidermis, not just stratum corneum. Pharm Res. 2013;30(4):1099-109.

33. Zorec B, Miklavčič D, Pavšelj N. Transdermal transport of calcein through electroporated skin-preliminary results of an ex vivo experiments. J Laser Health Acad. 2012;2012(1):S17.

34. Kocsis F, Bornatowicz N. "Ro 1525": Skin Sensitisation Study (BuehlerTest). Österreichisches Forschungszentrum Seibersdorf Ges.m.b.H., Seibersdorf, Austria, internal study code: HE47 sponsored by Henkel KGaA, Düsseldorf, Report No.R 9800963. 1998.

35. Goldin A, Beckman JA, Schmidt AM, Creager MA. Advanced glycation end products sparking the development of diabetic vascular injury. Circulation. 2006;114:597-605.

36. Daoud S, Schinzel R, Neumann A, Loske C, Fraccarollo D, Diez $\mathrm{C}$, et al. Advanced glycation endproducts: activators of cardiac remodeling in primary fibroblasts from adult rat hearts. Mol Med. 2001;7(8):543-51. 